\title{
Two-Photon Inner-Shell Ionization in the Extreme Ultraviolet
}

\author{
V. Richardson, ${ }^{1}$ J. T. Costello, ${ }^{1}$ D. Cubaynes, ${ }^{2}$ S. Düsterer, ${ }^{3}$ J. Feldhaus, ${ }^{3}$ H. W. van der Hart, ${ }^{4}$ P. Juranić,,${ }^{3}$ W. B. Li,,${ }^{3,5}$ \\ M. Meyer, ${ }^{2}$ M. Richter, ${ }^{6, *}$ A. A. Sorokin, ${ }^{3,6,7}$ and K. Tiedke ${ }^{3}$ \\ ${ }^{1}$ School of Physical Sciences and NCPST, Dublin City University, Dublin 9, Ireland \\ ${ }^{2}$ LIXAM/CNRS, Centre Universitaire Paris-Sud, Bâtiment 350, 91405 Orsay Cedex, France \\ ${ }^{3}$ Deutsches Elektronen-Synchrotron, DESY, Notkestraße 85, 22603 Hamburg, Germany \\ ${ }^{4}$ School of Mathematics and Physics, Queen's University Belfast, Belfast BT7 1NN, United Kingdom \\ ${ }^{5}$ Institute of Precision Optical Engineering, Department of Physics, Tongji University, 1239 SiPing Road, Shanghai 200092, China \\ ${ }^{6}$ Physikalisch-Technische Bundesanstalt, PTB, Abbestraße 2-12, 10587 Berlin, Germany \\ ${ }^{7}$ Ioffe Physico-Technical Institute, Polytekhnicheskaya 26, 194021 St. Petersburg, Russia
}

(Received 19 February 2010; published 29 June 2010)

\begin{abstract}
We have observed the simultaneous inner-shell absorption of two extreme-ultraviolet photons by a Xe atom in an experiment performed at the short-wavelength free electron laser facility FLASH. Photoelectron spectroscopy permitted us to unambiguously identify a feature resulting from the ionization of a single electron of the $4 d$ subshell of Xe by two photons each of energy $(93 \pm 1) \mathrm{eV}$. The feature's intensity has a quadratic dependence on the pulse energy. The results are discussed and interpreted within the framework of recent results of ion spectroscopy experiments of Xe obtained at ultrahigh irradiance in the extreme-ultraviolet regime.
\end{abstract}

DOI: 10.1103/PhysRevLett.105.013001

PACS numbers: $32.80 . \mathrm{Rm}, 32.80 . \mathrm{Fb}, 32.80 . \mathrm{Hd}, 42.50 . \mathrm{Hz}$

The advent of high-intensity extreme-ultraviolet (EUV) and $\mathrm{x}$-ray free electron lasers (FELs) has heralded a new era in the study of nonlinear optical processes. These facilities put this well established area into a new domain where the optical field exhibits a high degree of coherence in addition to an unprecedented combination of high average and peak intensity at high photon energy. As a result, inner-shell electrons and, indeed, highly correlated multielectron excited states can become important mediators of the multiphoton-matter interaction. Since it began operation in 2005, the EUV Free Electron Laser in Hamburg (FLASH) [1] has hosted a wide range of investigations in atomic and molecular physics including experiments on dilute targets such as molecular ions and highly charged ion beams, few-photon few-electron ionization processes, two-color coherent processes, and ultrafast pump-probe experiments $[2,3]$.

The simplest nonlinear process one can drive in an intense EUV laser field is two-photon ionization. Consequently, it is a prototypical process and its pursuit has become an important benchmark experiment for the study of the response of the simplest quantum systems, ranging from atoms to clusters, to intense high-frequency electromagnetic fields. Some years ago, high-order harmonic sources developed to the point where they could attain the threshold intensities needed to drive two-photon processes in an atom. The very first results were reported in breakthrough experiments at the very limit of intensities that can be obtained with these high-order harmonic sources. These included two-photon single and double ionization of $\mathrm{He}$ [4-6] and single ionization of the valence shells of Ar and Xe [7]. However, in focused beams,
FLASH yields irradiance levels which can approach $10^{16} \mathrm{~W} \mathrm{~cm}^{-2}$ [8], and so it provides a platform for a dramatic expansion of the range of sequential and simultaneous multiphoton ionization experiments that could be performed at EUV wavelengths. A good example is an experiment where FLASH permitted the full kinematics of two-photon double ionization of the valence shell of $\mathrm{Ne}$ to be recorded by using the cold target recoil ion momentum spectroscopy technique [9].

Exceptional behavior of the light-matter interaction at high irradiance has been demonstrated in two recent rare gas photoionization studies $[8,10]$ employing ion spectroscopy at FLASH in the EUV spectral range. At irradiance levels of a few $10^{15} \mathrm{~W} \mathrm{~cm}^{-2}$, nearly 50 EUV photons of $90 \mathrm{eV}$ photon energy interact within 10-20 fs with a single $\mathrm{Xe}$ atom and lead to the emission of up to 21 electrons. Notably, under the same experimental conditions, $\mathrm{Ne}, \mathrm{Ar}$, and $\mathrm{Kr}$ do not show this high degree of multiple photoionization. The experimental evidence suggests that the large one-photon cross section offered by the $4 d \rightarrow \varepsilon f$ giant resonance remains at the heart of the unique response of Xe to intense EUV fields. This resonance is a unique feature of Xe in the EUV and arises in the photon energy range from about 85 to $115 \mathrm{eV}$ [11]. For many years, it has represented a prime example of the impact of electron correlation on inner-shell photoionization at low irradiance $[12,13]$. The correlations may be described by a collective motion of the ten $4 d$ electrons, driven by the oscillation field of the electromagnetic wave, which results in the emission of one of their members. The effective radial potential for the emitted $\varepsilon f$ electron shows a biwell structure [14]. The potential barrier between the inner and the 
outer region of the atom explains the delayed onset of the $4 d$ one-photon ionization leading to the strong and broad one-photon $4 d \rightarrow \varepsilon f$ resonance feature, known as the giant resonance. In the case of $\mathrm{Xe}$, it is centered $30 \mathrm{eV}$ above the $4 d$ ionization threshold and dominates most of the $4 d$ oscillator strength. As pointed out in Refs. [8,15], lowcharge ion stages may be formed in one-photon $4 d \rightarrow \varepsilon f$ ionization of Xe followed by Auger decays providing the platform for subsequent multiphoton ionization of the ions. In this work, we wish to determine if the strength and the nature of the $4 d \rightarrow \varepsilon f$ resonance may open up, at high irradiance, additional ionization channels, namely, the simultaneous multiphoton excitation from the inner $4 d$ shell. In order to begin to answer this question and to obtain a broader insight into the photoionization of Xe at high EUV irradiance levels beyond $10^{15} \mathrm{~W} \mathrm{~cm}^{-2}$, we have initiated an investigation of the underlying processes by using electron spectroscopy.

Figure 1 illustrates the salient features of the experimental setup. It is essentially a combination of setups described in Refs. $[8,10,16]$. The photon energy of $(93 \pm 1) \mathrm{eV}$ was chosen as it permitted us to employ a high-quality industrial grade spherical $\mathrm{Si} / \mathrm{Mo}$ multilayer mirror [17] of the type used in EUV-lithography systems to focus the FEL beam diameter close to $3 \mu \mathrm{m}$. The pulse energy was measured on a shot-to-shot basis by using a gas-monitor detector [18]. An irradiance of up to $10^{16} \mathrm{~W} \mathrm{~cm}^{-2}$ could be achieved in the focus. The Xe gas filled the experimental vacuum chamber homogeneously at low pressure $\left(\sim 10^{-4} \mathrm{~Pa}\right)$. Emitted electrons were analyzed by using a magnetic bottle-type electron time-of-flight (TOF) spectrometer [16]. A retardation voltage was applied across the entrance to the TOF analyzer to prevent electrons with kinetic energies below a given threshold from entering the flight tube. A beam block in front of the mirror enabled us to determine the signal produced by the unfocused incoming beam (which had a diameter of a few millimeters) and to extract the electron spectrum corresponding only to the strongly focused FEL beam. To account for the background signal, all single-shot spectra were first sorted

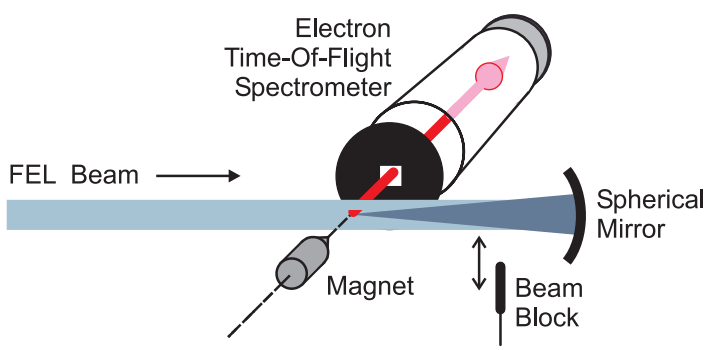

FIG. 1 (color online). Experimental setup: a magnetic bottletype electron spectrometer was used for the electron analysis. Focusing of the FEL beam was achieved by a spherical multilayer mirror. Spectra were taken with the unfocused and the unfocused plus focused FEL beam in the chamber by using a beam block in front of the mirror. according to the intensity of the gas-monitor detector signal. The intensity-appropriate backgrounds were then subtracted.

One-photon ionization is the dominant process for the unfocused beam and/or for low intensities of the FEL beam. Part of the corresponding electron spectrum is displayed in Fig. 2 showing the $4 d$ photolines at kinetic energies of 25.5 and $23.5 \mathrm{eV}$ which correspond to ionization of the $4 d_{5 / 2}^{-1}$ and $4 d_{3 / 2}^{-1}$ spin-orbit components, respectively. The corresponding $\mathrm{N}_{45} \mathrm{O}_{23} \mathrm{O}_{23}$ Auger lines appear in the kinetic energy region extending from 29 to $38 \mathrm{eV}$. The discrete features observed in high resolution Auger spectra $[19,20]$ are blended mainly into two asymmetric features around 32 and $35 \mathrm{eV}$ as a result of the resolution limit of the magnetic bottle spectrometer.

An additional broad and asymmetric line appears in electron spectra recorded at higher FLASH irradiance, of the order of $10^{16} \mathrm{~W} \mathrm{~cm}^{-2}$ in the focus, centered at a kinetic energy of about $118 \mathrm{eV}$ as shown in Fig. 3. We set a retarding field of $-90 \mathrm{~V}$ at the entrance to the TOF for this series of spectra, so that photoelectrons produced by one-photon processes were rejected and electron signals obtained were due solely to multiphoton processes. Spectra were recorded with and without Xe gas in the chamber to allow the determination of the background contribution from residual gas and scattered light. In addition, the spectra recorded show only a very small contribution from the second harmonic of the FEL radiation when the spherical mirror was blocked.

In order to conclusively confirm the observation of a multiphoton process, the integrated intensity of the broad

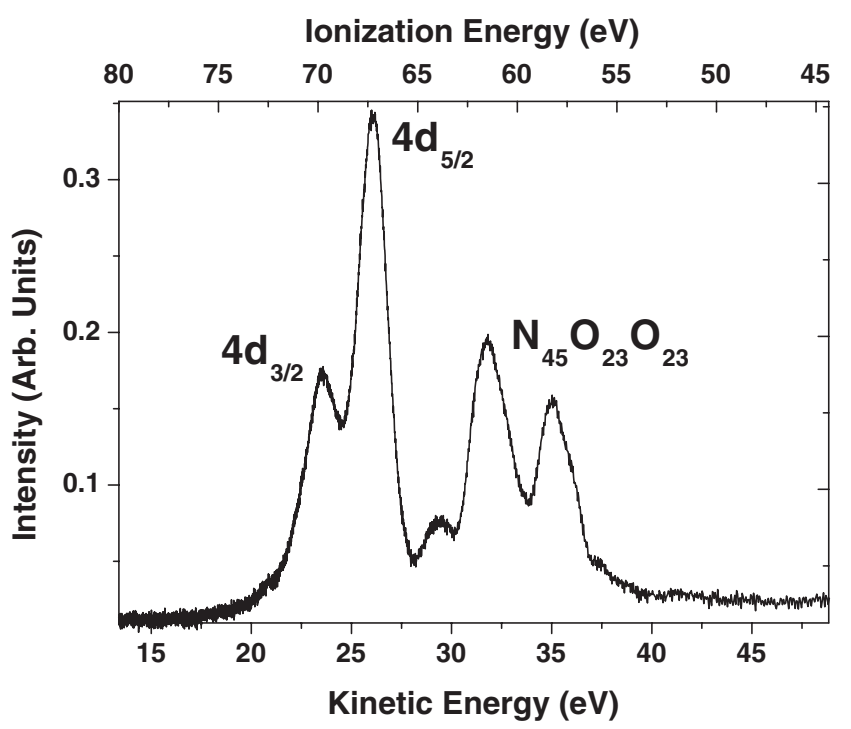

FIG. 2. Electron spectrum produced by the interaction of FLASH operating at the photon energy of $(93 \pm 1) \mathrm{eV}$ with a $\mathrm{Xe}$ gas target. The data were obtained at the lowest irradiance used in the experiment, of the order of $10^{10} \mathrm{~W} \mathrm{~cm}^{-2}$. The spectrum is dominated by the one-photon $4 d$ ionization and the subsequent $\mathrm{N}_{45} \mathrm{O}_{23} \mathrm{O}_{23}$ Auger decay. 


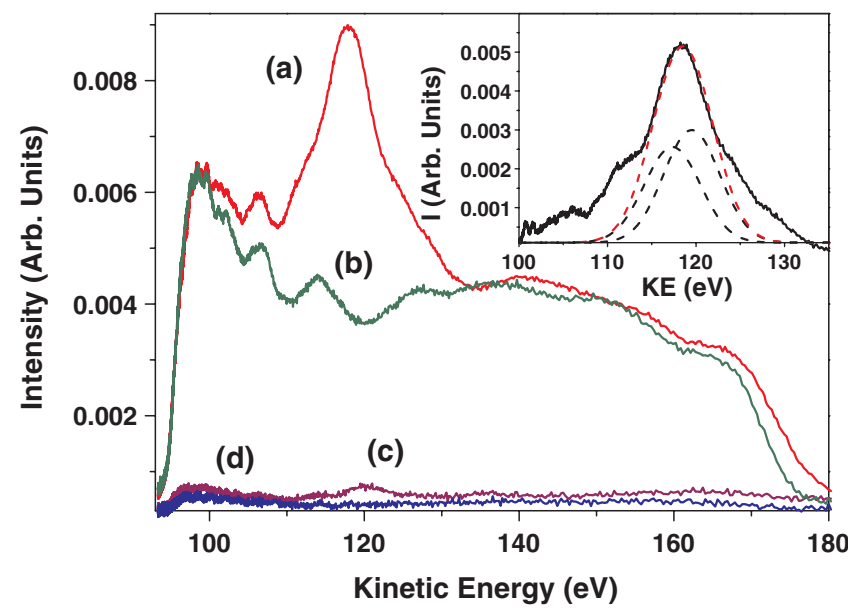

FIG. 3 (color online). Electron spectra recorded at the highest FLASH irradiance of about $10^{16} \mathrm{~W} \mathrm{~cm}^{-2}$ in the focus. The photon energy was $(93 \pm 1) \mathrm{eV}$. Spectra recorded under different experimental conditions are shown: (a) focused plus unfocused FEL interacting with Xe, (b) focused and unfocused FEL interacting with residual gas, (c) unfocused FEL interacting with $\mathrm{Xe}$, and (d) unfocused FEL interacting with residual gas. The inset shows the pure Xe spectrum due to the focused FEL, i.e., the difference spectrum (a) $-(b)-(c)+(d)$, fitted by the sum of two Gaussians separated by $2 \mathrm{eV}$.

line at about $118 \mathrm{eV}$ kinetic energy in Fig. 3 was measured as a function of the FEL pulse energy. The result is shown in Fig. 4 on a double-logarithmic scale. According to perturbation theory, the quadratic dependence, as evidenced by the slope of $1.95 \pm 0.20$, confirms that a twophoton process is the cause of the $118-\mathrm{eV}$ feature. In the case of a photoline, the corresponding ionization energy results in $2 \times(93 \pm 1)-(118 \pm 2)=(68 \pm 3) \mathrm{eV}$,

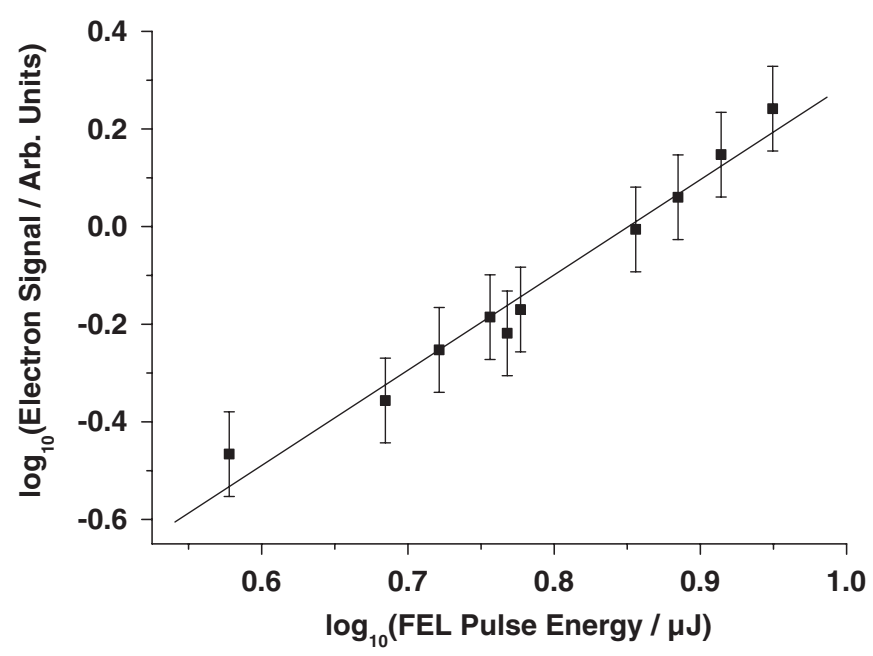

FIG. 4. Electron yield of the $118-\mathrm{eV}$ feature in Fig. 3 as a function of pulse energy. The slope of the linear fit is $1.95 \pm 0.20$ and confirms that the feature arises from a two-photon ionization process. which fits well with the $4 d$ ionization energy range of atomic Xe (Fig. 2). The experimental bandwidth seems to be, however, much broader, which we explain by space charge effects [21]: From ion spectroscopy results obtained on $\mathrm{Xe}$ at $93 \mathrm{eV}$ and at irradiance levels in the vicinity of $10^{16} \mathrm{~W} \mathrm{~cm}^{-2}$ [8], one may conclude that the FEL field produces and interacts with a highly ionized target. Because of the latter, moreover, electron emissions also from Xe ions have to be taken into consideration. $\mathrm{Xe}^{+}$as a target has four $4 d^{-1}$ ionization thresholds at 71.6, 72.9, 74.9 , and $76.2 \mathrm{eV}$ [22], which would yield photolines in the kinetic energy range from 110 to $115 \mathrm{eV}$. However, $\mathrm{Xe}^{+}$, in particular, appears only weakly in the ion spectra even at very high FEL intensity [8]. More highly charged ions appear in much greater numbers but yield electrons with kinetic energies below $100 \mathrm{eV}$ due to the increased $4 d^{-1}$ ionization energies for doubly and more highly charged Xe ions. $O$-shell ionization, e.g., $5 p^{-1}$ ionization of $\mathrm{Xe}^{5+}$ with an ionization energy at $67 \mathrm{eV}$ or $5 s^{-1}$ ionization of $\mathrm{Xe}^{4+}$ with an ionization energy at $69 \mathrm{eV}$ [23], could produce photoelectrons with relevant kinetic energies but the cross sections for these subshells are expected to be much weaker than for the $4 d$ case. We note also that, for atomic $\mathrm{Xe}$, there may be a contribution from $4 p^{-1}$ Auger processes [24] to the low energy wing of the broad feature at $118 \mathrm{eV}$ in Fig. 3. However, even at the highest FEL intensities we did not find any clear signatures in our spectra of a $4 p^{-1}$ ionization process, which would require two photons of $93 \mathrm{eV}$ energy and would produce photoelectrons in the $40-45 \mathrm{eV}$ range. Thus, we conclude that the $118-\mathrm{eV}$ feature in Fig. 3 is mainly due to the twophoton $4 d^{-1}$ ionization process in atomic Xe.

In order to test whether the $4 d \rightarrow \varepsilon f$ giant resonance has any effect on the two-photon $4 d^{-1}$ process, we have carried out calculations by using the $R$-matrix Floquet approach [25]. The residual $\mathrm{Xe}^{+}$states are described by using HF orbitals only [26]. The Xe atom is described by combining the allowed final $\mathrm{Xe}^{+}$states with basis functions for the continuum electron. The allowed Xe photoionization processes include emission of a $4 p, 4 d, 5 s$, or $5 p$ electron. The Floquet expansion contained 7 blocks of which 4 describe absorption with angular momenta up to $L=5$. Because of the limited amount of atomic structure included, the calculations are not very accurate. For example, the $4 d$ or $4 p$ binding energies obtained differ from the experimental values by about 5 and $14 \mathrm{eV}$, respectively. Nevertheless, the calculations give first insights into the physics of the two-photon absorption process. They confirm the large overlap between the confined $4 d$ orbital and the $\varepsilon f$ wave function well above threshold in the presence of the biwell potential for the excited $\varepsilon f$ electron [14]. Our calculations show, moreover, that, at the estimated experimental mean irradiance levels (in the focus) of about $10^{16} \mathrm{~W} \mathrm{~cm}^{-2}$, the two-photon emission of a $4 d$ electron via an $\varepsilon f$ state accounts for at least $0.5 \%$ of the total photoionization 
rate. The experimental ratio is, however, smaller due to our large interaction volume and the considerable detection of one-photon emissions from outside the focus.

According to our calculations, the two-photon $4 d^{-1}$ ionization process favors emission of $g$ electrons. Since the absorption of the first photon is theoretically confirmed to be enhanced by the $4 d$ giant resonance, our two-photon $4 d^{-1}$ ionization process proceeds as a sort of sequence:

$$
4 d \rightarrow \varepsilon f \rightarrow \varepsilon^{\prime} g
$$

Because of the first $4 d \rightarrow \varepsilon f$ step, the scheme in Eq. (1) represents a resonant process. Because of the continuum character of the intermediate $\varepsilon f$ electron state, it resembles, however, direct rather than sequential two-photon ionization. The observation of this so-called abovethreshold ionization process, thus, demonstrates that inner-shell ionization may play a significant role in nonlinear photoionization in the EUV. The large one-photon ionization cross section offered by the $4 d \rightarrow \varepsilon f$ giant resonance leads to an enhanced cross section also of the two-photon above-threshold ionization. In order to explain, at $10^{16} \mathrm{~W} \mathrm{~cm}^{-2}$ in the EUV, the observation of high charge states on Xe, simultaneous multiphoton ionization of many $4 d$ electrons was proposed $[8,10]$. By our electron spectroscopy experiments, however, direct multiple instead of just only single $4 d$ ionization could not be detected. On direct multiple ionization processes, the electrons emitted share their energy. It leads to a continuous background in the spectra which is, however, masked by the much more dominant atomic one-photon single electron emissions as shown in Fig. 2 and so cannot be distinguished.

In conclusion, we have studied the two-photon $4 d$ innershell ionization of Xe in a focused EUV beam at FLASH by electron spectroscopy. The corresponding photoelectron line in Fig. 3 represents the first detection of an abovethreshold ionization two-photon process in an inner electron shell. Our work demonstrates the significance of inner shells and inner-shell resonances in nonlinear multiphotonmatter interaction for experiments at the new EUV and $\mathrm{x}-$ ray laser facilities.

We thank the FLASH team for a very successful FEL operation. This work was supported by the Deutsche Forschungsgemeinschaft under Grant No. DFG RI 804/51 and by Science Foundation Ireland Grant No. 07/IN.1/
I1771, IRCSET, and the European Framework Programme FP7 Grant No. 226716.

*mathias.richter@ptb.de

[1] W. Ackermann et al., Nat. Photon. 1, 336 (2007).

[2] J. T. Costello, J. Phys. Conf. Ser. 88, 012057 (2007).

[3] C. Bostedt et al., Nucl. Instrum. Methods Phys. Res., Sect. A 601, 108 (2009).

[4] Y. Kobayashi, T. Sekikawa, Y. Nabekawa, and S. Watanabe, Opt. Lett. 23, 64 (1998).

[5] N. A. Papadogiannis et al., Phys. Rev. Lett. 90, 133902 (2003).

[6] Y. Nabekawa et al., Phys. Rev. Lett. 94, 043001 (2005).

[7] N. Miyamoto et al., Phys. Rev. Lett. 93, 083903 (2004).

[8] A. A. Sorokin et al., Phys. Rev. Lett. 99, 213002 (2007).

[9] A. Rudenko et al., Phys. Rev. Lett. 101, 073003 (2008).

[10] M. Richter et al., Phys. Rev. Lett. 102, 163002 (2009).

[11] VUV and Soft X-Ray Photoionization, edited by U. Becker and D. A. Shirley (Plenum, New York, 1996), and references therein.

[12] Giant Resonances in Atoms, Molecules, and Solids, edited by J.P. Connerade, J.M. Esteva, and R. C. Karnatak (Plenum, New York, 1987), and references therein.

[13] M. Ya. Amusia and J. P. Connerade, Rep. Prog. Phys. 63, 41 (2000).

[14] Z. Crljen and G. Wendin, Phys. Rev. A 35, 1571 (1987).

[15] M. G. Makris, P. Lambropoulos, and A. Mihelič, Phys. Rev. Lett. 102, 033002 (2009).

[16] P. Radcliffe et al., Nucl. Instrum. Methods Phys. Res., Sect. A 583, 516 (2007).

[17] T. Feigl, S. Yulin, N. Benoit, and N. Kaiser, Microelectron. Eng. 83, 703 (2006).

[18] K. Tiedtke et al., J. Appl. Phys. 103, 094511 (2008).

[19] W. Yates, K. H. Tan, L. L. Coatsworth, and G. M. Bancroft, Phys. Rev. A 31, 1529 (1985).

[20] H. Aksela et al., Phys. Rev. A 33, 3867 (1986).

[21] A. Sorokin et al., Appl. Phys. Lett. 89, 221114 (2006).

[22] A. Gottwald, Ch. Gerth, and M. Richter, Phys. Rev. Lett. 82, 2068 (1999).

[23] J.-M. Bizau et al., Phys. Rev. A 73, 022718 (2006).

[24] S. Heinasmaki et al., J. Electron Spectrosc. Relat. Phenom. 137-140, 281 (2004).

[25] P. G. Burke, P. Francken, and C. J. Joachain, J. Phys. B 24, 761 (1991).

[26] E. Clementi and C. Roetti, At. Data Nucl. Data Tables 14, 177 (1974). 